\title{
Left atrial dissection after mitral operation mimicking severe mitral regurgitation
}

\author{
Martin C. Heidt, MD, Ares K. Menon, MD, Peter Roth, MD, Zan Mitrev, MD, Hakan K. Akintürk, MD, and \\ Paul R. Vogt, MD, FETCS, FICA, Giessen, Germany
}

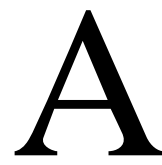

67-year-old patient underwent mitral valve reconstruction consisting of quadrangular resection at the P2 segment and implantation of a flexible 29-mm Duran annuloplasty ring (Medtronic, Inc, Minneapolis, Minn). Five days after surgery, transesophageal echocardiography (TEE) revealed left atrial dissection that was causing severe paravalvular regurgitation because of a reentry in the left atrial dissection membrane. The mitral leaflets still demonstrated appropriate coaptation within the annuloplasty ring.

\section{Clinical Summary}

This patient presented with dyspnea and was in New York Heart Association class III. Echocardiography revealed degenerative mitral valve disease with mitral regurgitation grade 3 resulting from chordal rupture and subsequent posterior leaflet prolapse at the P2 segment. The mitral valve annulus was enlarged. The left ventricular ejection fraction was $75 \%$, and the diastolic diameter was 60 $\mathrm{mm}$. The left atrial diameter was $45 \mathrm{~mm}$. Coronary angiography results were normal.

Mitral reconstruction was performed. This consisted of quadrangular resection of the prolapsing P2 segment, annular reconstruction with 2 annular compression sutures at the site of the leaflet resection, leaflet reconstruction with single sutures, and a ring annuloplasty with a 29-mm Duran ring. Intraoperative TEE demonstrated residual mitral regurgitation grade 1 or less. The early postoperative course was uneventful. On the fifth postoperative day, the patient complained of dyspnea. Bilateral pleural effusion was confirmed by chest radiograph. Echocardiography showed suspected recurrent mitral regurgitation (grade 4). Finally, TEE (Figures 1 and 2) revealed partial dehiscence of the annuloplasty ring at the P3 segment. The endocardium was still attached to the annuloplasty ring; however, there was a severe paraannular mitral regurgitation (grade 4) between the left atrial endocardium and the left atrial myocardium (Figure 1, arrow 1). Blood flow into the false left atrial lumen entered the true left atrium through a hole in the endocardial layer, serving as reentry and preventing left

\footnotetext{
From the Department of Cardiovascular Surgery, University Hospital, Giessen, Germany.

Received for publication July 21, 2003; revisions requested Sept 11, 2003; revisions received Sept 16, 2003; accepted for publication Oct 8, 2003.

Address for reprints: Martin C. Heidt, MD, Department of Cardiovascular Surgery, University Hospital Giessen, Rudolf-Buchheim-St 7, D-35392 Giessen, Germany (E-mail: Martin.C.Heidt@innere.med.uni-giessen.de).

J Thorac Cardiovasc Surg 2004;127:596-7

$0022-5223 / \$ 30.00$

Copyright (C) 2004 by The American Association for Thoracic Surgery doi:10.1016/j.jtcvs.2003.09.030
}

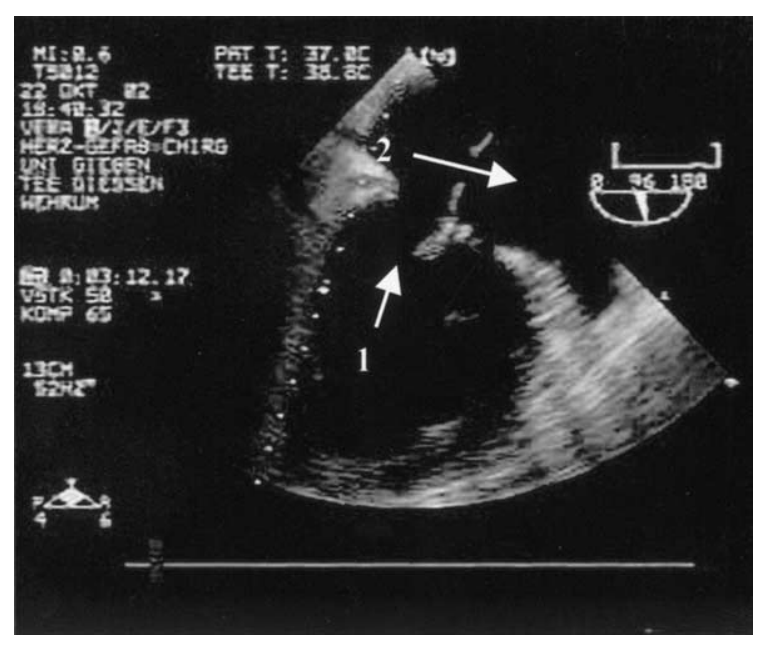

Figure 1. Entry to the false lumen (arrow 1). Connection from the false lumen to the left atrium (arrow 2).

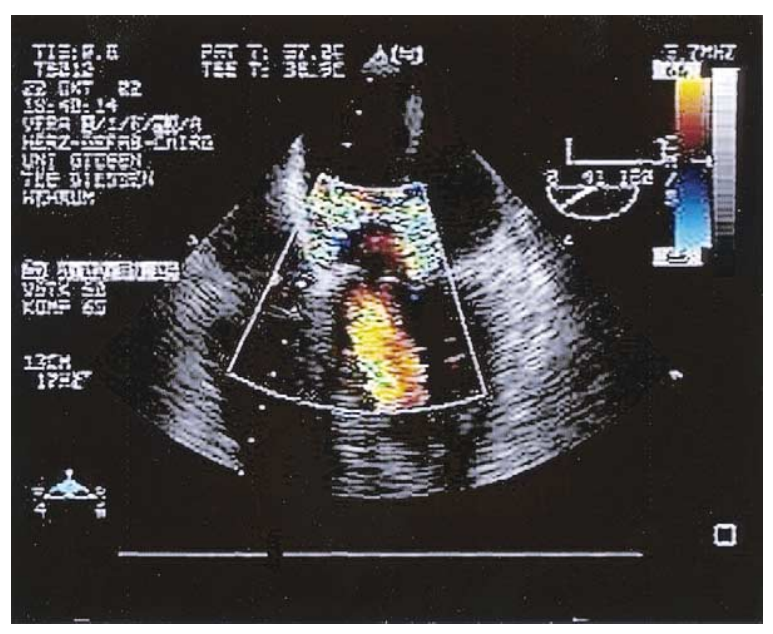

Figure 2. Color Doppler image with flow from the left ventricular cavity to the false lumen and back to the left atrium.

atrial compression caused by an intramural, intramyocardial hematoma usually found in this setting (Figure 1, arrow 2). The left atrial endocardium remained attached to the entire circumference of the prosthesis, producing 2 left atrial cavities connected through the reentry as described. The leaflet reconstruction within the ring annuloplasty was intact; hence, leaflet coaptation within the ring was appropriate. Pulmonary artery pressure was estimated by echocardiography to be increased to $60 \mathrm{~mm} \mathrm{Hg}$. 
Reoperation confirmed the described TEE findings demonstrating ring dehiscence around the $\mathrm{P} 3$ segment, the endocardium being attached to the annuloplasty ring, and left atrial dissection, as well as the reentry into the true atrial lumen. There were no epicardial hematomas and no signs of atrioventricular disconnection or subepicardial bleeding.

The left atrial endocardium, being the dissection membrane, was glued to the myocardium, obliterating the false left atrial lumen. The atrial reentry was sutured. A mechanical prosthesis was implanted after reconstruction of the posterior mitral annulus with 2 autologous pericardial strips, creating a stable mitral annulus around the P3 segment and the posterior commissure. Intraoperative and postoperative TEE demonstrated a mitral prosthesis without any signs of residual dissection or paraprosthetic leakage. The postoperative course was uneventful aside from a protracted weaning from mechanical ventilation.

\section{Discussion}

Left atrial dissection after mitral valve operation occurs in approximately $0.8 \%$ of cases. ${ }^{1}$ Mostly the dissection forms a large cavity between the left atrial endocardium and myocardium, showing suffusions leading to a massive intramural hematoma that finally compresses the cavity of the left atrium. ${ }^{2}$ In these cases, mitral regurgitation is not the leading sign. Instead, a left atrial pseudoaneurysm can be found early or late after surgery. ${ }^{3}$ In our patient, early recurrent mitral regurgitation after reconstruction was suspected by transthoracic echocardiography performed for early postoperative recurrence of dyspnea and bilateral pleural effusion 5 days after uneventful mitral valve reconstruction.

TEE detected subendocardial paravalvular regurgitation at the P3 segment, the blood flowing from the left ventricular cavity back into a false lumen between the left atrial myocardium and left atrial endocardium. The endocardium remained attached to the annuloplasty ring. The false left atrial lumen was decompressed due to a reentry within the left atrial endocardium, directing the blood again toward the reconstructed mitral valve within the annuloplasty ring, functionally completing mitral regurgitation.

With this, the regurgitant portion of the blood coming from the left ventricle completed the mitral regurgitation as, in systole, the blood crossed the reentry and the reconstructed mitral valve through the normally coapting mitral leaflet within the ring annuloplasty. The ring dehiscence may be due to tissue friability in a patient with a floppy mitral valve, probably associated with the use of a flexible ring instead a flexible posterior annuloplasty, possibly preventing dehiscence by a more smooth adaptation to the geometry of the mitral annulus. ${ }^{4}$ However, there are no data recommending flexible annuloplasty rings to prevent left atrial dissection.

Left atrial dissection is a rare complication after mitral reconstruction. Paravalvular mitral regurgitation with or without left atrial compression due to a left atrial pseudoaneurysm is considered a key echocardiographic finding.

\section{References}

1. Martinez-Selles M, Garcia-Fernandez MA, Moreno M, Bermejo J, Delcan JL. Echocardiographic features of left atrial dissection. Eur J Echocardiogr. 2000;1:147-50.

2. Genoni M, Jenni R, Schmid ER, Vogt PR, Turina MI. Treatment of left atrial dissection after mitral repair: internal drainage. Ann Thorac Surg. 1999;68:1394-6.

3. Idir M, Deville C, Roudaut R. Delayed left atrial wall dissection after mitral valve replacement. Echocardiography. 2000;17:259-61.

4. Ninomiya M, Taketani T, Ohtsuka T, Motomura N, Takamoto S. A rare type of left atrial dissection. J Thorac Cardiovasc Surg. 2002;124:61820.

5. Gallego P, Oliver JM, Gonzalez A, Dominguez FJ, Sanchez-Recalde A, Mesa JM. Left atrial dissection: pathogenesis, clinical course, and transesophageal echocardiographic recognition. J Am Soc Echocardiogr. 2001;14:813-20. 